\title{
Total oxidation of naphthalene at low temperatures using palladium nanoparticles supported on inorganic oxide-coated cordierite honeycomb monoliths
}

\author{
Francisco J. Varela-Gandía ${ }^{a}$, Ángel Berenguer-Murcia ${ }^{a}$, Dolores Lozano-Castelló ${ }^{a}$, Diego Cazorla- \\ ${ }_{5}$ Amorós $^{a^{*}}$, David R. Sellick ${ }^{b}$, Stuart H. Taylor ${ }^{b^{*}}$ \\ Received (in XXX, XXX) Xth XXXXXXXXX 20XX, Accepted Xth XXXXXXXXX 20XX \\ DOI: 10.1039/b000000x
}

A study on the preparation of thin films of ZSM-5 and BETA zeolites, and a SAPO-5 silicoaluminophosphate, supported on cordierite honeycomb monoliths by in situ synthesis was carried 10 out for their use as catalyst supports. Furthermore $\gamma-\mathrm{Al}_{2} \mathrm{O}_{3}$ was also coated onto a cordierite honeycomb monolith by a dip-coating method for use as a standard support. Structured monolithic catalysts were prepared by impregnation of the aforementioned coated monoliths with polymer-protected Pd nanoparticles. The monolithic catalysts have been tested for the total oxidation of naphthalene (100 ppm, GHSV $1220 \mathrm{~h}^{-1}$ ). From the combined use of the zeolite with polymer-protected nanoparticles, enhanced 15 catalytic properties have been found for the total abatement of naphthalene. The Pd/MBETA and Pd/MZSM-5 catalytic monoliths have shown excellent activity with a high degree of stability, even after undergoing accelerated ageing experiments.

\section{Introduction}

Polycyclic aromatic hydrocarbons (PAHs), such as naphthalene, 20 are environmentally hazardous compounds produced as a result of the incomplete combustion or pyrolysis of organic material [15]. Naphthalene is considered the least toxic and simplest molecule of the PAHs, and thus it is used as a model compound for this group of pollutants. In the literature, a range of different 25 technologies have been studied to reduce PAH emissions [6-13]. Among them, catalytic combustion is the most promising for the removal of PAHs from polluted air streams, due to its lower operating temperature and higher selectivity towards $\mathrm{CO}_{2}[2]$.

For catalytic total oxidation, the types of catalysts used are based 30 to a large extent on metals such as Pd, Pt, Ru or Co supported on $\gamma$-alumina by impregnation [14], metal oxide catalysts [2,15-19], mesoporous aluminosilicates with supported Pt [20] or the use of zeolites ion-exchanged with Pt [21]. Based on our previous studies [22], the use of polymer protected Pd nanoparticles 35 supported on zeolites is one of the most promising types of catalyst for the total oxidation of naphthalene, due to the low oxidation temperature reported $\left(165-180^{\circ} \mathrm{C}\right)$, and the high stability of the catalyst.

However, for practical applications macro-structured catalysts are ${ }_{40}$ preferred over powdered catalysts. For example, a monolithic reactor can involve a single block of material containing a large number of parallel channels, which are available in various sizes or shapes [23]. Low pressure drops, uniform flow distribution, absence of hot spots and good tolerance to plugging by dust are ${ }_{45}$ essential requisites that ultimately lead to the use of catalytic monoliths [23,24]. For gas phase applications, metal or ceramic honeycomb monoliths are standard support materials. In reference to ceramic monoliths, the support structure is made of a non-catalytic, thermally resistant material, typically cordierite, 50 onto which a catalytic layer is deposited [25]. The chemical composition of the cordierite is based on $2 \mathrm{MgO} \cdot 2 \mathrm{Al}_{2} \mathrm{O}_{3} \cdot 5 \mathrm{SiO}_{2}$ and a cordierite monolith has excellent operational properties such as low pressure drops in the exhaust system, good thermal resistance, refractoriness, good wash-coat adherence and 55 compatibility between wash-coat and catalyst [26,27]. Concerning the deposition method for active phases, different strategies can be employed for initially coating the monolith channels with a support material, like alumina, silica, ceria and zeolites, followed by subsequent impregnation with an active ${ }_{60}$ phase [28]. In the case of zeolites or SAPO materials, there are two ways to coat the monoliths: hydrothermal synthesis using either direct synthesis or seeded growth, referred to as in situ synthesis, and dip-coating or wash-coating, which consists of deposition from a slurry of zeolite particles followed by a ${ }_{65}$ stabilizing thermal treatment [24].

In the present study, two different zeolites (BETA and ZSM-5) and a silicoaluminophosphate molecular sieve (SAPO-5) have been selected as coating materials for cordierite monoliths by in situ synthesis. Furthermore, a commercial $\gamma-\mathrm{Al}_{2} \mathrm{O}_{3}$ has been 70 coated on the monolithic supports by the dip-coating method for comparative purposes. The active phase that will be employed is polymer protected Pd nanoparticles, which have shown good properties as catalysts for naphthalene total oxidation with the supports above, when they were used as powder catalysts [22]. ${ }_{75}$ Special attention has focused on the stability and recyclability of 
the structured catalysts for extended time-on-stream experiments.

\section{Experimental}

\section{Catalyst preparation}

Coating monoliths with BETA, ZSM-5, and SAPO-5

${ }_{5}$ Cylindrical cordierite monoliths (Corning) were used as supports (400 cpsi, length (l): $14 \mathrm{~cm}$, diameter (d): $14 \mathrm{~cm}$ ). These were cut into small cylindrical pieces (l: $1.6 \mathrm{~cm}$, d: $1.4 \mathrm{~cm}$, mass: $\sim 1.2 \mathrm{~g}$ ). Prior to coating, all the small monoliths were blown with air to remove the dust produced in the cutting process. A calcination ${ }_{10}$ process in static air in a furnace at $800^{\circ} \mathrm{C}$ for 2 hours (heating rate: $6{ }^{\circ} \mathrm{C} / \mathrm{min}$ ) was performed with the aim to remove organic impurities.

The growth of BETA and ZSM-5 zeolites and SAPO-5 silicoaluminophosphate molecular sieve was carried out by in15 situ synthesis onto the cordierite monolith avoiding the use of binders. The general experimental procedure for the synthesis of the zeolites and the silicoaluminophosphate onto the monolith was:

i. The cordierite honeycomb monoliths were wrapped with 20 Teflon tape to prevent zeolite growth on the outer surface.

ii. The "teflonated" monoliths were placed in a stainless steel autoclave with a Teflon liner (V: $10 \mathrm{~mL}$ ). A precursor gel/monolith weight ratio of $4 \mathrm{~mL} / \mathrm{g}$ was used for coating the monoliths.

25 iii. The autoclaves were placed horizontally in a convection oven and rotated during the whole growth process $(\mathrm{v}=4 \mathrm{rpm})$ to ensure a homogeneous growth of the solid film and prevent blocking of the monolith channels [27]. Four monoliths were prepared per batch.

30 iv. The crystallization step was carried out at the desired temperature and time for each crystalline material.

v. After the hydrothermal synthesis, the autoclaves were submitted to "Fast cooling" using running tap water.

vi. After the synthetic protocol, the coated monoliths were

35 washed with abundant quantities of distilled water and dried at $100{ }^{\circ} \mathrm{C}$ overnight. All the monoliths were submitted to sonication for 6 hours to remove loosely adhered crystals and then, dried again overnight in an oven at $100{ }^{\circ} \mathrm{C}$.

Two consecutive synthesis steps were performed. Thus, after 40 finishing the whole procedure described above the samples were again placed into an autoclave for a second synthesis process using a fresh synthesis solution (i.e. points (2) to (6) were repeated).

After preparing the coated monoliths using two consecutive 45 syntheses, the removal of the template was carried out by calcination at the desired temperature for each material in a muffle furnace under static air (heating rate $1{ }^{\circ} \mathrm{C} / \mathrm{min}$ ). Finally, the total weight increase for both the first coating step performed and the whole coating process was measured. The increase in 50 mass is indicative of the loading and is expressed as wt\%.

Coating of the monoliths with zeolite BETA was carried out following the methodology reported by Bueno-Lopez et al. [27], but it was adapted to our larger monolith size. The details of the molar compositions of the synthesis gel are given in [19]. The 55 optimum conditions found for the synthesis of zeolite BETA on cordierite monoliths were two consecutive synthesis steps with a crystallization temperature of $132^{\circ} \mathrm{C}$ for 48 hours. The BETA coated monoliths, named MBETA, were submitted to a calcination step to remove the template at $500^{\circ} \mathrm{C}$ for 6 hours.

60 ZSM-5 coated monoliths, which are called MZSM-5 in this work, were obtained after two consecutive synthesis steps. As mentioned previously by Ulla et al. [25], the coating of high aluminium content zeolites is hindered by Al-rich supports, such as cordierite, and therefore it is necessary to combine two 65 synthesis protocols using materials with different $\mathrm{Si} / \mathrm{Al}$ ratios. For this reason, the first-crystallization step was the preparation of a silicalite-1 coating, which is isostructural to ZSM-5, but with no aluminium content in its framework, in order to ensure the crystallization and anchoring of the ZSM-5 zeolite onto the 70 monolith. Thus, the synthesis was performed adapting the methodology for the preparation of silicalite-1/carbon membranes reported by Berenguer-Murcia et al. [29]. The silicalite-1 solution was obtained by adding 3.420g of TEOS (tetraethoxysilane) dropwise into a $1 \mathrm{M}$ Tetrapropylammonium hydroxide TPA-OH

75 solution (4.920 g TPA-OH solution, in $31.570 \mathrm{~g}$ of distilled water). The resulting solution was aged for $90 \mathrm{~min}$ and the monoliths were coated with a layer of silicalite- 1 after a crystallization time of 6 hours at $180^{\circ} \mathrm{C}$. The second crystallization step consisted of the preparation of a ZSM-5 80 zeolite coating. The details of the experimental procedure used for the preparation of ZSM-5 zeolite are given in detail elsewhere [30]. According to reference [30], the crystallization conditions are $190^{\circ} \mathrm{C}$ for 10 hours. Template removal was carried out by calcination in static air at $550{ }^{\circ} \mathrm{C}$ for 4 hours.

${ }_{85}$ SAPO-5 monoliths, named MSAPO-5, were prepared by adapting the procedure described by Campelo et al. [31] for the preparation of powdered SAPO-5. The crystallization was performed at $200^{\circ} \mathrm{C}$ and crystallization times between 18 and 24 hours were used for studying the coating process. The calcination 90 process for MSAPO- 5 monoliths was performed at $600^{\circ} \mathrm{C}$ for 6 hours.

Coating of $\gamma-\mathrm{Al}_{2} \mathrm{O}_{3}$

A coating of $\gamma-\mathrm{Al}_{2} \mathrm{O}_{3}$ onto selected honeycomb cordierite monoliths was performed by dip-coating and the samples were 95 labeled as $\mathrm{M}-\boldsymbol{\gamma}-\mathrm{Al}_{2} \mathrm{O}_{3}$. Prior to the dip-coating step, the cordierite monoliths were wrapped with Teflon tape to avoid deposition of $\gamma-\mathrm{Al}_{2} \mathrm{O}_{3}$ on their outer surface. $\mathrm{M}-\gamma-\mathrm{Al}_{2} \mathrm{O}_{3}$ monoliths were prepared following the procedure described by Villegas et al. [32]. The $\gamma-\mathrm{Al}_{2} \mathrm{O}_{3}$ powder was dispersed in $\mathrm{HNO}_{3}\left(\gamma-\mathrm{Al}_{2} \mathrm{O}_{3} / \mathrm{H}_{2} \mathrm{O}=\right.$ 10025 wt. \%, $\mathrm{HNO}_{3} / \gamma-\mathrm{Al}_{2} \mathrm{O}_{3}=2 \mathrm{mmol} / \mathrm{g}$ ) using a high-shear mixer (ULTRA TURRAX T25, IKA Labortechnik). Initially, the required amount of $\gamma-\mathrm{Al}_{2} \mathrm{O}_{3}$ powder was added to the acid solution for $10 \mathrm{~min}$ at $7000 \mathrm{rpm}$. After vigorous stirring (14000 rpm) for $5 \mathrm{~min}$ at room temperature, the stable suspension was 105 used for dip-coating. A series of 8 monoliths were prepared with each dip-coating suspension, in order to check the reproducibility of the coated monoliths. The monoliths were immersed vertically into the suspension for 2 minutes and the excess suspension was removed using a flow of compressed air. Finally, the monoliths 110 were dried at room temperature for 24 hours whilst continuously rotating horizontally to ensure optimum coating distribution. The procedure was performed twice with a fresh suspension. M- $\gamma$ $\mathrm{Al}_{2} \mathrm{O}_{3}$ monoliths were calcined in static air at $600{ }^{\circ} \mathrm{C}$ for 4 hours (heating rate $1^{\circ} \mathrm{C} / \mathrm{min}$ ).

115 Deposition of Pd nanoparticles

Palladium nanoparticles protected by polyvinylpirrolidone (PVP) 
were synthesized by the reduction-by-solvent method as reported previously [33]. The catalysts used in this work were prepared by the impregnation method, as reported in our previous work for powdered catalysts [22]. The aforementioned coated monoliths 5 were wrapped with Teflon tape in order to fix them vertically in $10 \mathrm{~mL}$ Teflon liners and to prevent deposition of Pd nanoparticles on the outer surface of the cordierite monolith. The required concentration of Pd nanoparticles, suspended in methanol, was added based on the total amount of BETA, ZSM-5, SAPO-5 or $\gamma$ $10 \mathrm{Al}_{2} \mathrm{O}_{3}$ coating, to yield a nominal 1 wt.\% of metallic loading with respect to the zeolite, SAPO-5 or $\mathrm{Al}_{2} \mathrm{O}_{3}$. To ensure the complete immersion of the coated monoliths in the suspension additional methanol was added to give a final volume of $5 \mathrm{ml}$. The Teflon liners filled with the monolith and Pd suspension were placed in a 15 thermostated bath, stirred at $60 \mathrm{rpm}$ at room temperature for two days. Subsequently, they were transferred to an oven, where they were left at $60{ }^{\circ} \mathrm{C}$ in order to evaporate the solvent and obtain the final structured honeycomb monolith, labeled Pd/MBETA, $\mathrm{Pd} / \mathrm{MZSM}-5$, $\mathrm{Pd} / \mathrm{MSAPO}-5$ and $\mathrm{Pd} / \mathrm{M} \gamma-\mathrm{Al}_{2} \mathrm{O}_{3}$.

\section{${ }_{20}$ Characterization of the catalysts}

ZSM-5 and BETA zeolites and SAPO-5 silicoaluminophosphate molecular sieve supported on cordierite monoliths were characterised by XRD, using a 2002 Seifert powder diffractometer. The scanning rate was $2 \% \mathrm{~min}$ and $\mathrm{Cu}-\mathrm{K} \alpha$ 25 radiation was used.

The coated monoliths were characterized by SEM in a JEOL microscope (model JSM-80). The monoliths were carefully cut parallel to the monolith axis. This procedure allowed the analysis of the surface of the monolith channels, and the thickness and 30 homogeneity of the coated layer. In the case of the Pd-containing catalysts, they were cut in the same manner and analyzed by SEM in a HITACHI S-3000 microscope. Energy-dispersive X-ray spectrometry (EDX) was used to ascertain the Pd concentration along the channels by careful cutting of the monolith along its 35 length.

Textural characterization of the coated monoliths and the catalysts was carried out by means of adsorption of $\mathrm{N}_{2}$ at $-196{ }^{\circ} \mathrm{C}$ (Autosorb 6, Quantachrome). Prior to the adsorption measurements, the samples were outgassed under vacuum $\left(10^{-2}\right.$ $40 \mathrm{mbar}$ ) at $250{ }^{\circ} \mathrm{C}$ for $4 \mathrm{~h}$ to remove any adsorbed impurities. Surface area was calculated from nitrogen adsorption isotherms using the BET equation $\left(S_{\mathrm{BET}}\right)$. Total microporevolumes $\left(V_{\mathrm{DR}}\right.$ $\left(\mathrm{N}_{2}\right)$ ) were calculated by applying the Dubinin-Radushkevich (DR) equation to the $\mathrm{N}_{2}$ adsorption data at $-196^{\circ} \mathrm{C}$ [34].

\section{${ }_{45}$ Catalyst performance}

Catalytic activity tests for naphthalene oxidation were carried out in a fixed bed reactor (diameter $=1.6 \mathrm{~cm}$ ). The feed stream consisted, in all cases, of 100ppmv naphthalene in a mixture of $20 \% \mathrm{O}_{2}$ and $80 \%$ He. The total flow was set to $50 \mathrm{ml} / \mathrm{min}$ (GHSV $\left.{ }_{50}=1220 \mathrm{~h}^{-1}\right)$. Analysis of reactants and reaction products was performed by on-line gas chromatography using thermal conductivity and flame ionization detectors. The catalytic activity was measured over the temperature range $100-200^{\circ} \mathrm{C}$ in incremental steps and temperatures were measured by a 55 thermocouple placed in the catalyst bed connected to a PID controller. Data were collected at each temperature after a stabilization time of 20 minutes. Three analyses were made at each temperature to ensure steady state was attained. Oxidation activity is expressed as a yield of carbon dioxide. Furthermore, 60 accelerated time-on-line experiments for long-term use of these catalysts were performed with the aim to determine potential deactivation. For this purpose, the catalysts were submitted to reaction conditions at $250^{\circ} \mathrm{C}$ for 48 hours.

\section{Results and discussion}

\section{${ }_{65}$ Coated monoliths characterization}

Table 1 shows the percentage of weight increase on coated monoliths after the first and second coating steps. According to Bueno-Lopez et al. [27] in order to improve zeolite loading and deposit a thin and homogeneous zeolite layer, autoclave rotation 70 during the hydrothermal treatment and a second synthesis step are necessary. It is worth mentioning that both the supported zeolite and SAPO-5 crystals (after the first step) and the zeolite and SAPO-5 layer (after the second step) are highly stable towards sonication, indicating good adhesion of the zeolite to the 75 monoliths surface. Therefore, the error in the estimation of the weight increase after two coating steps is reasonably low, considering the complex process under study, including crystallization, cleaning and calcinations steps. $\mathrm{M}-\gamma-\mathrm{Al}_{2} \mathrm{O}_{3}$ monoliths, prepared in batches of 8 monoliths at a time, possess a 80 high reproducibility with a weight increase and standard deviation similar to observations by Villegas et al. [32].

Table 1 Monolith weight increase (wt\%) for single and two steps coatings.

\begin{tabular}{ccc}
\hline Coated monolith & $\begin{array}{c}\text { First coating step } \\
\text { (wt.\%) }\end{array}$ & $\begin{array}{c}\text { Second coating step } \\
\text { (wt.\%) }\end{array}$ \\
MBEA & $3.6 \pm 0.4$ & $17.7 \pm 2.0$ \\
MZSM-5 & $4.0 \pm 0.4$ & $15.8 \pm 1.8$ \\
$\mathrm{MSAPO}-5$ & $4.9 \pm 0.9$ & $16.1 \pm 1.7$ \\
$\mathrm{M} \gamma-\mathrm{Al}_{2} \mathrm{O}_{3}$ & $9.4 \pm 1.4$ & $15.0 \pm 1.7$
\end{tabular}

${ }_{85}$ X-ray diffraction was used to confirm the phase purity and crystallinity of the zeolite or SAPO layers coated on the cordierite monoliths after two synthesis steps. Figure 1 compiles the diffractograms of uncoated cordierite and coated monoliths (MBETA, MZSM-5 and MSAPO-5). Powder zeolites and SAPO90 diffractograms have been added to confirm the crystallinity of the coating layer; the diffractogram for the coated monoliths show the same characteristic peaks of the powdered zeolites (indicated by full dots on the corresponding peaks) [35]. Therefore, it can be confirmed that the crystallinity of the zeolites 95 are maintained when they are supported on the monoliths.

In the case of MSAPO-5 monoliths, a synthesis time between 18$24 \mathrm{~h}$ was used. From the analysis of the XRD of the samples prepared after 18, 20, 22 and 24h (results not shown), it was confirmed that only the MSAPO-5 prepared after 18h (XRD 100 included in Figure 1) showed the characteristic peaks of the SAPO-5 silicoaluminophosphate (AFI structure) without the appearance of other crystalline phases. Consequently, all the monoliths synthesized for this work were prepared with a synthesis time of $18 \mathrm{~h}$. 


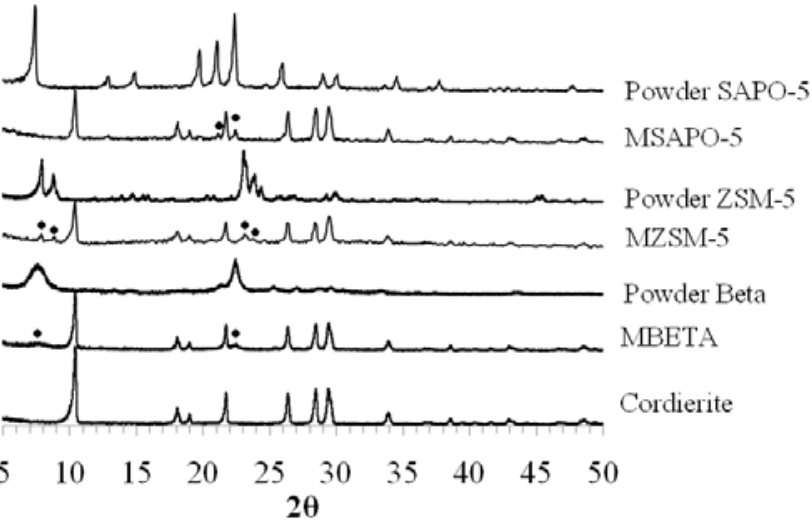

Fig. 1 XRD diffractograms of cordierite, coated monoliths and powder zeolites. The peaks corresponding to the zeolite phases are marked with full dots

5 Nitrogen adsorption was performed to analyze the porous texture of the as-prepared materials. From the nitrogen isotherms (see Figure 2) it is possible to confirm that cordierite exhibits negligible porosity; therefore, it can be assumed that there is no contribution of the cordierite support to the adsorption properties 10 of the coated monoliths. Table 2 summarizes the surface areas $\left(S_{\mathrm{BET}}\right)$ and the total micropore volumes $\left(V_{\mathrm{DR}}\left(\mathrm{N}_{2}\right)\right)$ calculated per unit weight of the monolith.

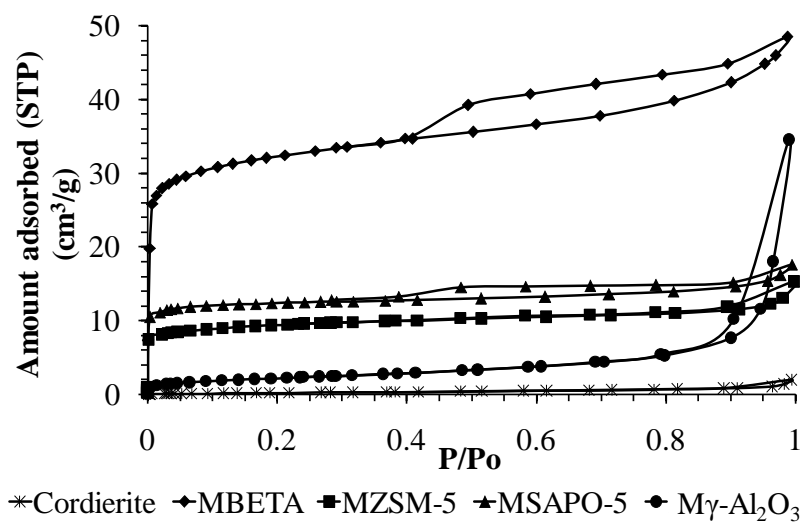

Fig. 2 Nitrogen adsorption/desorption isotherms at $-196{ }^{\circ} \mathrm{C}$

15 Table 2 Porous texture characterization results of the coated monoliths

$\begin{array}{ccc}\text { Sample } & \boldsymbol{S}_{\text {BET }}\left(\mathbf{m}^{2} / \mathbf{g}\right) & \boldsymbol{V}_{\text {DR }}\left(\mathbf{N}_{\mathbf{2}}\right)\left(\mathbf{c m}^{3} / \mathbf{g}\right) \\ \text { Cordierite } & 1 & 0.00 \\ \text { MBETA } & 100 & 0.05 \\ \text { MZSM-5 } & 35 & 0.02 \\ \mathrm{MSAPO}-5 \gamma-\mathrm{Al}_{2} \mathrm{O}_{3} & 35 & 0.02 \\ & 8 & -{ }^{\mathrm{a}}\end{array}$

${ }^{a}$ Not possible to determine.

In the case of $\mathrm{M} \gamma-\mathrm{Al}_{2} \mathrm{O}_{3}$ it presents a similar nitrogen adsorption isotherm as the powder $\gamma-\mathrm{Al}_{2} \mathrm{O}_{3}$ used to coat the monolith, which indicates that the dip-coating procedure used to prepare this 20 sample does not modify significantly the porosity of the supported $\gamma-\mathrm{Al}_{2} \mathrm{O}_{3}$.

In reference to zeolite and SAPO coated materials, MBETA, MZSM-5 and MSAPO-5, the nitrogen adsorption isotherms also have the same shape, including the hysteresis loop, as the

25 equivalent powdered samples prepared in previous work [35]. To compare the specific surface area $\left(S_{\mathrm{BET}}\right)$ area and micropore volume of the coated zeolites with the powdered zeolites, estimations for the monoliths have been made considering the weight of the zeolite coatings. These values have been excluded 30 in this paper for brevity, but it is evident that both BETA and SAPO-5 layers have very similar porosity to the powder materials, thus practically they are unaffected by the in situ coating process.

On the other hand, the ZSM-5 layer, confirmed by X-ray 35 diffraction, shows a lower porosity than the one expected from the comparison with a powder sample. The main reason is due to the coating procedure used for this sample. As we have mentioned previously, the coating of high aluminium content zeolites is hindered by Al-rich supports such as cordierite.

40 According to Ulla et al. [25] the first step in the hydrothermal synthesis of zeolite films is the formation of a precursor gel layer onto the substrate, which serves as the primary source of nucleation. However, the addition of $\mathrm{Al}$ to the solution accelerates gel layer formation, but delays the nucleation and ${ }_{45}$ subsequent zeolite growth processes within it. Therefore, the use of Al-rich synthesis gels has a direct influence on the kinetics of the process and, as a consequence, high loadings of wellcrystallized and anchored zeolite films are difficult to obtain. In a first synthesis, Silicalite- 1 is used to provide a suitable 50 environment for the growth of a high aluminium content zeolite in the second synthesis step. This first layer is partially dissolved during the second synthesis, helping the nucleation and growth of the second layer. Thus, this procedure may block or remove part of the first zeolite coating producing a reduction in the adsorption 55 properties of the zeolite layer [36].

A detailed study of the different coated layers onto the cordierite monoliths was carried out by SEM. Figure 3 shows the original uncoated monolith. The top view shows that a cordierite monolith is a continuous support with macroscopic openings along the ${ }_{60}$ entire surface that will be accessible during the zeolite coating process. From the cross sectional view it can be established that the monolith walls are $150 \mu \mathrm{m}$ wide and have a macroporous character.
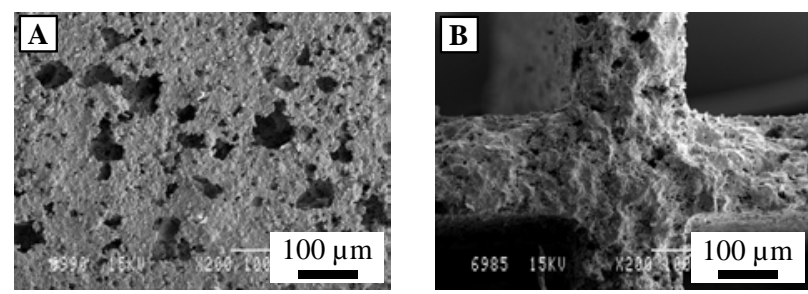

65 Fig. 3 SEM images of original uncoated cordierite honeycomb monolith (a) top view and (b) cross sectional view

Figure 4 shows the top view of the coated monoliths with BETA, ZSM-5, SAPO-5 and $\gamma-\mathrm{Al}_{2} \mathrm{O}_{3}$, respectively. After the first synthesis step (See Figure 4, left column), MBETA, MZSM-5 70 and MSAPO-5 monoliths have crystals which do not cover all the surface of the monoliths and, therefore, do not form a continuous layer. These individual crystals, however will act as seeds for the second in-situ synthesis step performed. The individual crystals formed onto the cordierite monoliths present the typical 75 morphology for each type of zeolite, as has been reported in the literature for BETA [27,35], ZSM-5 [29,35] and SAPO-5 [37,38]. In reference to the $\mathrm{M} \gamma-\mathrm{Al}_{2} \mathrm{O}_{3}$, the slurry for dip-coating partially 
covered the surface of the monoliths filling the external openings of the cordierite monoliths.
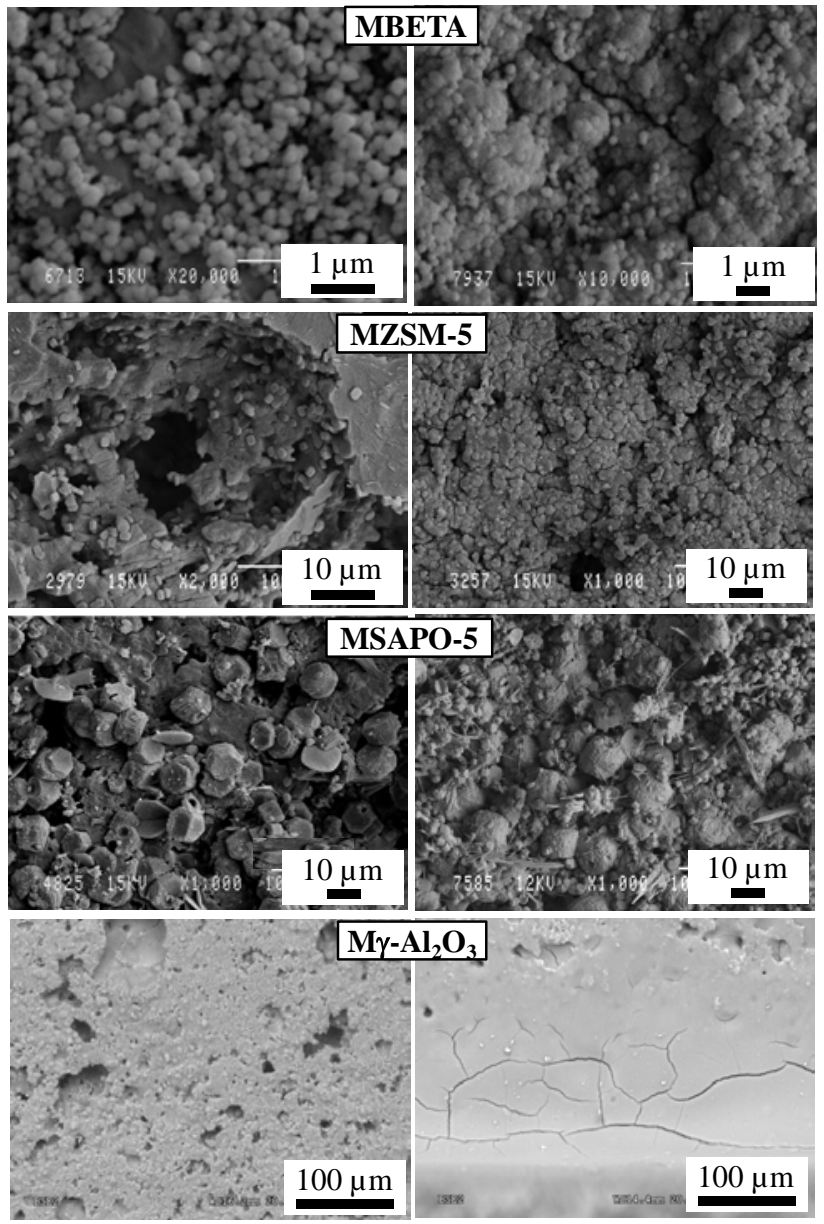

Fig. 4 SEM top view images for the four prepared monoliths after the first coating step (left column) and the second coating step (right column)

When a second synthesis step was performed (See Figure 4 right column), it is possible to cover all the surface of the monolith with a complete and homogeneous layer of intergrown crystals. MBETA and MZSM-5 crystals maintained their respective shape. 10 It must be pointed out that the MSAPO-5 monolith presents a bimodal particle size distribution. The crystals appearing after the first synthesis have increased in size up to approximately $12 \mu \mathrm{m}$, and small SAPO-5 crystals have grown (approximately $1 \mu \mathrm{m}$ ), filling the gaps between the larger crystals, giving rise to a 15 continuous layer. Finally, the $\mathrm{M} \gamma-\mathrm{Al}_{2} \mathrm{O}_{3}$ monolith prepared by the "dip-coating" method is formed by a homogeneous and continuous layer of $\mathrm{M} \gamma-\mathrm{Al}_{2} \mathrm{O}_{3}$ over the entire monolith wall. In spite of the deposited monolith coating layer being homogeneous, a significant quantity of $\gamma-\mathrm{Al}_{2} \mathrm{O}_{3}$ was preferentially deposited on 20 the corners towards the centre of the channels, and small cracks were observed after the calcination step.

With the aim to analyze the thickness of the coated layer onto the monolith walls, SEM images of the cross sectional view are useful for this purpose (see Figure 5). All samples in this study 25 prepared by the in situ synthesis method showed multiple layers of zeolite crystals. There were no significant differences between the coating zeolite or SAPO-5 layer in the centre and in the channel open ends, as well as along the channels, indicating that the movement of the autoclaves during synthesis results in a 30 homogeneous growth along the channel length.
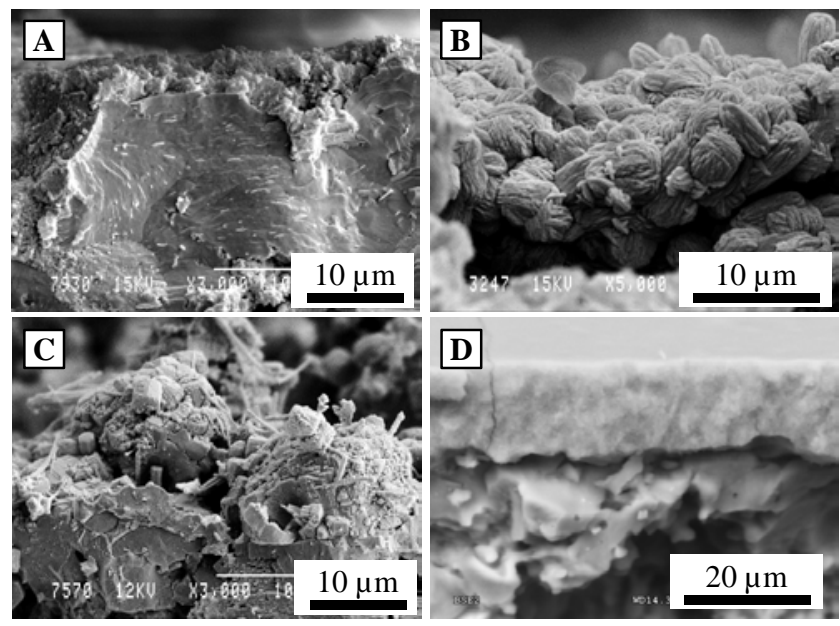

Fig. 5 SEM cross sectional images for the four prepared monoliths after the second coating step. (a) Sample MBETA, (b) Sample MZSM-5, (c) Sample MSAPO-5, and (d) Sample $\mathrm{M} \gamma-\mathrm{Al}_{2} \mathrm{O}_{3}$

35 Detailed analysis of SEM images has allowed us to conclude that the MBETA monolith (Figure 5a) is formed by a layer of intergrown zeolite crystals with an average thickness of $3 \mu \mathrm{m}$. Furthermore, the small crystal size of BETA zeolite permitted access to the internal openings of the monolith walls, thus giving 40 rise to a zeolite deposit formed by the intergrowth between the crystals in the internal and external surface, producing a three dimensional network of the zeolite layer that results in high mechanical stability [27]. Figure 6 shows, as an example, a cross sectional view of a monolith wall, where the BETA zeolite layer 45 was deposited on the cordierite wall, together with an internal opening filled with zeolite crystals.

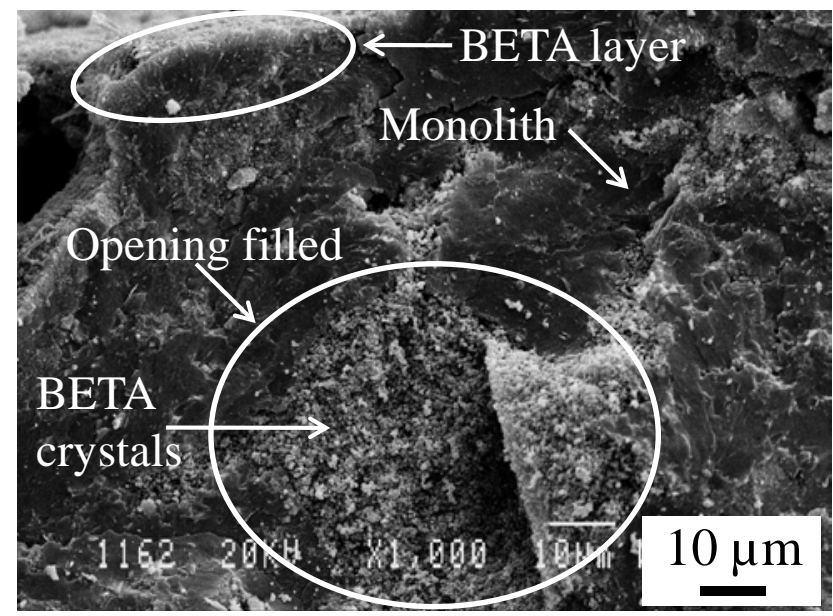

Fig. 6 SEM cross sectional images for BETA zeolite monolith with an internal opening completely filled with BETA zeolite

50 In the case of MZSM-5 monoliths, the coating layer consists of intergrown crystals with an average thickness of $6 \mu \mathrm{m}$. It is worth mentioning that the previous Silicalite- 1 crystals deposited during the first synthesis cannot be observed. As explained previously, these crystals act as seeds for the deposition of a stable ZSM-5 55 layer and are partially dissolved during hydrothermal synthesis. MSAPO-5 monoliths are formed by a coated layer of intergrown 
crystals with a bimodal particle size distribution with a layer thickness of around $12 \mu \mathrm{m}$. To conclude, for the monoliths prepared by in situ synthesis, it is important to note that MZSM-5 and MSAPO-5 only exhibit the crystallization of individual 5 zeolite crystals in the internal openings without filling the porosity within the monolith walls, contrary to the behaviour observed for the MBETA monolith.

Analyzing the $\gamma-\mathrm{Al}_{2} \mathrm{O}_{3}$ layer on $\mathrm{M} \gamma-\mathrm{Al}_{2} \mathrm{O}_{3}$ monoliths (See Figure 5), a variation in thickness has been found. The thickness of the 10 alumina wash-coating varies from 3-6 $\mu \mathrm{m}$ on the left side of the monolith to $16 \mu \mathrm{m}$ on the other end of the opening, whilst it was about $12 \mu \mathrm{m}$ in the middle of the channel as determined by SEM. This behaviour can be attributed to the immersion/extraction rate of the monolith from the slurry and the final step of blowing with 15 air that may alter the coated layer at one of the ends.

\section{Monolithic catalysts characterization}

After impregnation, the monolithic catalysts were analyzed by XRD and no appreciable changes were observed in the prepared catalysts (results not shown).To analyze the distribution and 20 loading of Pd nanoparticles on the monolith, Energy-dispersive X-ray spectrometry (EDX) was used to measure the quantity of Pd along the channels of the monolith. From the individual analysis performed on the four catalysts (Pd/MBETA, $\mathrm{Pd} / \mathrm{MZSM}-5, \quad \mathrm{Pd} / \mathrm{SAPO}-5$ and $\mathrm{Pd} / \mathrm{M} \gamma-\mathrm{Al}_{2} \mathrm{O}_{3}$ ), the general 25 conclusion was that the amount of $\mathrm{Pd}$ in all the monoliths was around $1 \mathrm{wt} \%$ with respect to the coating layer and the Pd was uniformly distributed along the channels.

Nitrogen adsorption was performed on the Pd-monolith catalysts in the same manner as the coated monoliths. Table 3 includes the 30 BET surface areas $\left(S_{\mathrm{BET}}\right)$ and the total micropore volumes $\left(V_{\mathrm{DR}}\left(\mathrm{N}_{2}\right)\right)$. Comparing the results with those obtained for the coated monoliths (see Table 2), it can be established that the catalyst preparation method has affected the adsorption properties of the coated layer on the Pd/MBETA and Pd/MZSM-5 and ${ }_{35} \mathrm{Pd} / \mathrm{M} \gamma-\mathrm{Al}_{2} \mathrm{O}_{3}$ only to a minor extent. Only in the case of the Pd/MSAPO-5 catalyst was there a significant reduction of the $\mathrm{N}_{2}$ adsorption capacity compared to the MSAPO-5 monolith before impregnation with $\mathrm{Pd}$ nanoparticles. From the $\mathrm{N}_{2}$ adsorption isotherms (results not shown), it is possible to confirm that, after

40 impregnation with the Pd-based nanoparticles, the shape of the isotherms is the same as for the coated monoliths.

Table 3 Porous texture characterization results of the catalytic monoliths

\begin{tabular}{ccc}
\hline Sample & $\boldsymbol{S}_{\mathbf{B E T}}\left(\mathbf{m}^{2} / \mathbf{g}\right)$ & $\boldsymbol{V}_{\mathbf{D R}}\left(\mathbf{N}_{\mathbf{2}}\right) \mathbf{( \mathbf { c m } ^ { 3 } / \mathbf { g } )}$ \\
Pd/MBETA & 95 & 0.05 \\
Pd/MZSM-5 & 35 & 0.02 \\
Pd/MSAPO-5 & 10 & $-{ }^{\mathrm{a}}$ \\
Pd/Mץ-Al $\mathrm{O}_{3}$ & 7 & $-{ }^{-}$ \\
${ }^{a}$ Not possible to determine. & & \\
\hline
\end{tabular}

Comparing these results with powder catalysts Pd/BETA and ${ }_{45} \mathrm{Pd} / \mathrm{ZSM}$-5 prepared previously, [22], the decrease in $S_{\mathrm{BET}}$ is lower in the coated monoliths. This behavior could be tentatively assigned to the location of deposited Pd nanoparticles. When a powder catalyst is prepared, the Pd nanoparticles are located over all of the zeolite crystals homogeneously. On the contrary, Pd 50 nanoparticles in the coated monolith are predominantly distributed on the surface of the coating layer, due to the highly dense and compacted layers formed, as corroborated by EDX.
Similar to the Pd/SAPO-5 powdered catalyst [22], the catalytic monolith shows the same decrease in apparent surface area.

55 As mentioned in our previous work [22], the synthesized Pd colloid shows very low polydispersity with a particle size of $1.8 \pm$ $0.3 \mathrm{~nm}$. In the case of the prepared monoliths it is not possible to observe the Pd dispersion using TEM, but deposition of polymer protected Pd nanoparticles did result in a noticeable change of $\mathrm{Pd}$ 60 particle size according to our previous studies [22,33], so it is expected that the particle size increases when the polymer protected Pd nanoparticles are supported onto the coated monolith.

\section{Catalytic performance in the oxidation of naphthalene}

65 The catalytic activity of naphthalene oxidation was measured for the four monolithic catalysts. Three reaction cycles were performed with each catalyst in order to ensure the stability of the catalysts. This was achieved by incrementally increasing the reaction temperature to the desired set point and then cooling 70 down to room temperature in order to start the next catalytic cycle. Additionally, it is noteworthy that no CO was detected as a reaction product in any of the experiments performed. Previous to testing the monolithic $\mathrm{Pd}$ catalysts, the coated monoliths (MBETA, MZSM-5, MSAPO-5 and $\mathrm{M} \gamma-\mathrm{Al}_{2} \mathrm{O}_{3}$ ) were tested in 75 the oxidation of naphthalene and, in general, none of the coated monoliths was active at the temperature range studied (i.e. between 100 and $200{ }^{\circ} \mathrm{C}$ ). For example, the MBETA monolith was active at temperatures above $300{ }^{\circ} \mathrm{C}$ reaching a $45 \%$ conversion of naphthalene to $\mathrm{CO}_{2}$ at $350^{\circ} \mathrm{C}$.

${ }_{80}$ Figure 7 shows the variation of the catalytic activity (expressed as conversion towards $\mathrm{CO}_{2}$ ) for naphthalene oxidation, as a function of the reaction temperature for the four Pd-monolith catalysts. After the first cycle, outstanding catalytic performance has been observed for the $\mathrm{Pd} / \mathrm{M} \gamma-\mathrm{Al}_{2} \mathrm{O}_{3}$ and $\mathrm{Pd} / \mathrm{MSAPO}-5$ catalysts, whilst ${ }_{85} \mathrm{Pd} / \mathrm{MZSM}-5$ and Pd/MBETA were able to reach full naphthalene conversion at higher temperatures. It is worth mentioning that the catalytic activity remained unchanged after three cycles, maintaining high naphthalene oxidation activity to $\mathrm{CO}_{2}$ without any evidence for catalyst deactivation.

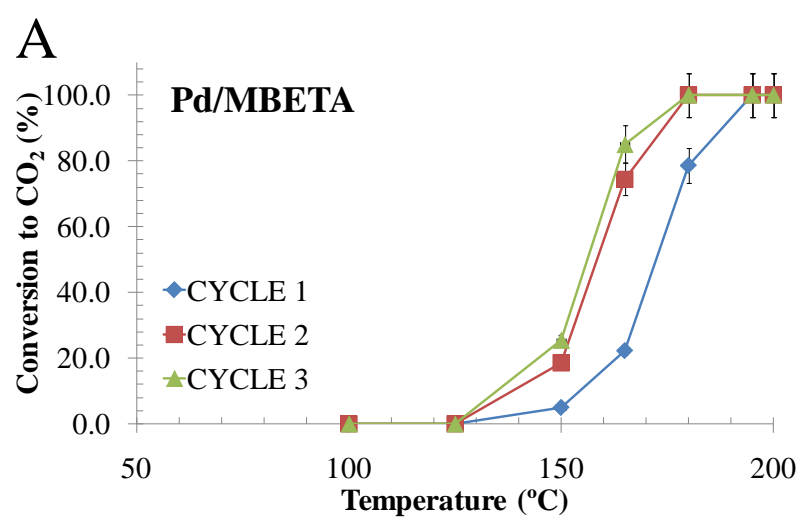

90

\footnotetext{
This journal is (C) The Royal Society of Chemistry [year] 

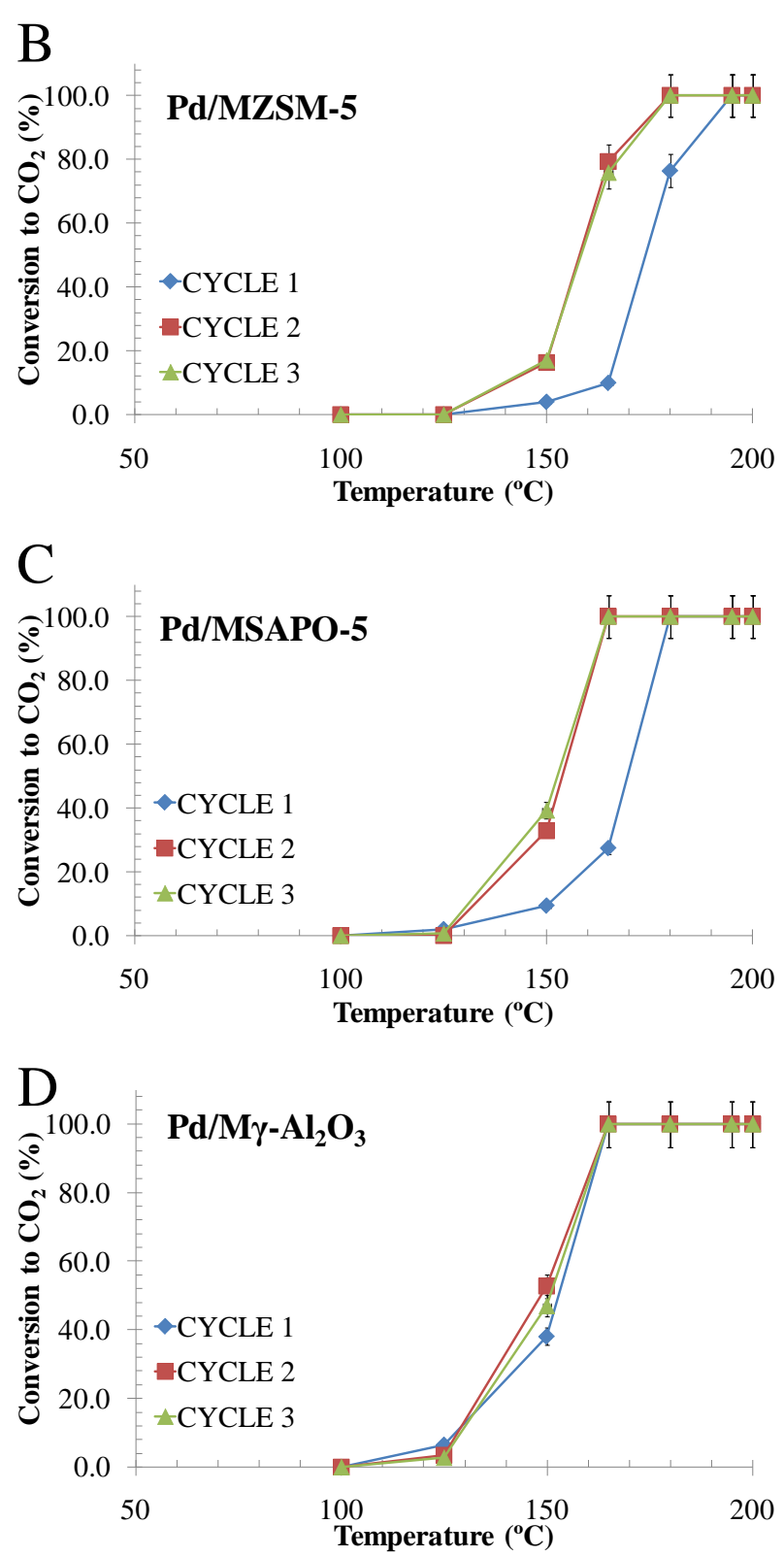

Fig. 7 Variation of the catalytic activity for naphthalene total oxidation (expressed as yield to $\mathrm{CO}_{2}$ ) as a function of reaction temperature over the four catalysts. (a) Pd/MBETA, (b) Pd/MZSM-5, (c) Pd/MSAPO-5, and

Table 4 shows the temperature required for total naphthalene oxidation to $\mathrm{CO}_{2}$ for each monolithic catalyst over the three cycles studied. Comparing these results with our previous work [14] a small increase in the temperature needed to reach total 10 oxidation is observed for the zeolite-coated monolith (BETA and ZSM-5). This can be due to the dense thin layer of the zeolite that may have an influence in naphthalene adsorption/desorption, thus having some effect on the kinetics of the process. Analyzing the $\mathrm{CO}_{2}$ conversion at $150^{\circ} \mathrm{C}$ in the monolith samples (see Table 5) 15 and comparing with our previous work [22], the catalytic activity of the monoliths at $150{ }^{\circ} \mathrm{C}$ shows a similar trend. The zeolite catalyst showed an increase of activity from the first to the third cycle, whilst the $\mathrm{Pd} / \mathrm{MAl}_{2} \mathrm{O}_{3}$ showed reduced activity after three cycles. The activity is slightly lower for the catalysts in monolith

20 form with respect to the powder form is slightly lower, and this is most probably due to increased diffusion limitations with the monoliths. Nevertheless, these are minor differences that show that the catalyst based on Pd nanoparticles supported on the coated-monoliths have a similar activity to the powder catalysts 25 [22].

Table 4 Temperature required for naphthalene total oxidation for the different catalysts for the three oxidation cycles tested.

\begin{tabular}{|c|c|c|c|}
\hline \multirow[b]{2}{*}{ Catalyst } & \multicolumn{3}{|c|}{ Temperature $\left({ }^{\circ} \mathrm{C}\right)$ for total conversion to $\mathrm{CO}$} \\
\hline & Cycle 1 & Cycle 2 & Cycle 3 \\
\hline 1\%Pd/MBETA & 195 & 180 & 180 \\
\hline 1\%Pd/MZSM-5 & 195 & 180 & 180 \\
\hline 1\%Pd/MSAPO-5 & 180 & 165 & 165 \\
\hline $1 \% \mathrm{Pd} / \mathrm{M} \gamma-\mathrm{Al}_{2} \mathrm{O}_{3}$ & 165 & 165 & 165 \\
\hline
\end{tabular}

It is also useful to start to probe the stability of the active 30 catalysts. An accelerated experiment to study possible deactivation as a function of time was conducted at $250^{\circ} \mathrm{C}$ for 48h. Figure 8 shows the time-on-line data for the four $\mathrm{Pd}$ monolith catalysts. It was evident that the Pd/MBETA and $\mathrm{Pd} / \mathrm{ZSM}-5$ samples were stable for at least $48 \mathrm{~h}$ and there is no 35 evidence of catalyst deactivation over the testing period. The yield of $\mathrm{CO}_{2}$ at the beginning of the experiment was $100 \%$ and it was not diminished after 48h. Contrary to these two catalysts, $\mathrm{Pd} / \mathrm{MSAPO}-5$ and $\mathrm{Pd} / \mathrm{M} \gamma-\mathrm{Al}_{2} \mathrm{O}_{3}$ exhibit a conversion of $100 \%$ during the first hours, but their conversion to $\mathrm{CO}_{2}$ dropped to ${ }_{40} 92 \%$ and $90 \%$ for $\mathrm{Pd} / \mathrm{MSAPO}-5$ and $\mathrm{Pd} / \mathrm{M} \gamma-\mathrm{Al}_{2} \mathrm{O}_{3}$, respectively, indicating possible deactivation, probably induced by the agglomeration of the Pd nanoparticles. Therefore, Pd/MBETA and $\mathrm{Pd} / \mathrm{MZSM}-5$ are the most suitable catalysts for naphthalene removal. In these cases, the rugosity of the prepared zeolite layer 45 prevents the agglomeration of the Pd nanoparticles. These observations are in agreement with those for the powder catalyst [22].

Table $5 \mathrm{CO}_{2}$ conversion at $150^{\circ} \mathrm{C}$ for the four catalysts and during the three cycles tested

\begin{tabular}{|c|c|c|c|}
\hline \multirow[b]{2}{*}{ Catalyst } & \multicolumn{3}{|c|}{$\mathrm{CO}_{2}$ conversion $(\%)\left(\mathrm{T}=150^{\circ} \mathrm{C}\right)$} \\
\hline & Cycle 1 & Cycle 2 & Cycle 3 \\
\hline 1\%Pd/MBETA & 5 & 19 & 25 \\
\hline 1\%Pd/MZSM-5 & 4 & 16 & 17 \\
\hline 1\%Pd/MSAPO-5 & 9 & 33 & 39 \\
\hline $1 \% \mathrm{Pd} / \mathrm{M} \gamma-\mathrm{Al}_{2} \mathrm{O}_{3}$ & 38 & 53 & 43 \\
\hline
\end{tabular}

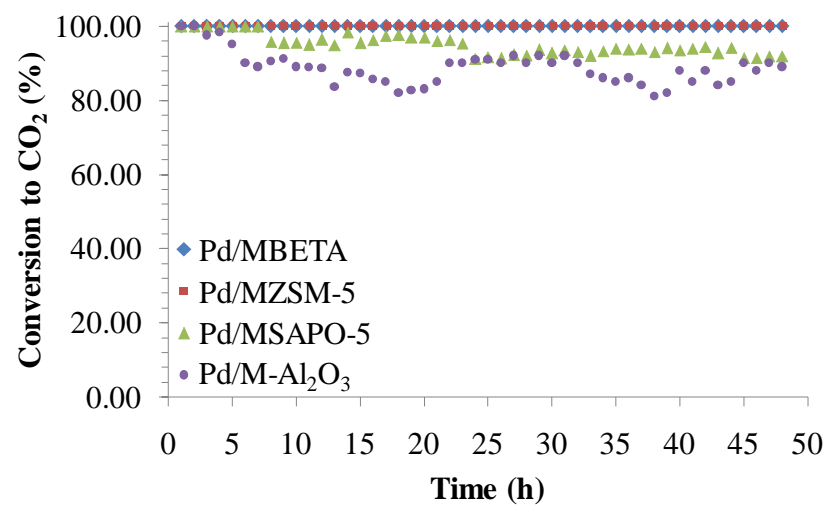

Fig. 8 Conversion of naphthalene to $\mathrm{CO}_{2}$ as a function of time-on-line at $250^{\circ} \mathrm{C}$ for the four monolithic catalysts 
These results help to assess the viability of the monolith-based catalysts for naphthalene removal by catalytic oxidation, but a critical comparison with monolith-based catalysts from the literature should also be considered. Neyestenaki et al. [21] 5 deposited ZSM-5 on cordierite monolith substrates by means of a ball-milled zeolite-water slurry method (monolith characteristics 400 cpsi, d=21.8 mm, h=20 mm), by dip-coating and drying several times, until a wash-coated loading between 13.5 and $30.8 \%$ and Pd, loaded by classical methods, was achieved. All the 10 monolithic catalysts were tested for the removal of the emissions of pollutants from the combustion of biofuels in a simulated mixture, which contained naphthalene (50ppm), methane, CO, $\mathrm{CO}_{2}$ and $\mathrm{O}_{2}$, with a balance of $\mathrm{N}_{2}\left(\mathrm{GHSV}=20000 \mathrm{~h}^{-1}\right)$. As expected, an increase in wash-coating loading resulted in 15 decreased light-off temperatures of all the pollutants. Focusing on the monolith with similar characteristics $(0.95 \% \mathrm{Pd} / \mathrm{NaZSM}-5)$ to our monolithic catalyst, the $\mathrm{T}_{50}$ was $246^{\circ} \mathrm{C}$ and $243^{\circ} \mathrm{C}$ for coated monoliths with an amount of ZSM-5 of $14.6 \%$ wt and $24.3 \%$ wt, respectively.

${ }_{20}$ Ferrandon et al. [39] studied the total oxidation of mixtures with different concentrations of the same aforementioned pollutants, including $50 \mathrm{ppm}$ of naphthalene (GHSV $=23000 \mathrm{~h}^{-1}$, catalyst volume $6.5 \mathrm{ml}$ ). The monoliths used were composed of cordierite honeycomb monoliths (400 cps) wash-coated with $\gamma-\mathrm{Al}_{2} \mathrm{O}_{3}$ and 25 used as supports for noble metal catalysts (Pd or Pt, $0.1 \mathrm{~mol} \%$ ), metal oxides $\left(\mathrm{MnO}_{\mathrm{x}} \mathrm{CuO}_{\mathrm{x}}, 10 \mathrm{~mol} \%\right)$ and combinations of noble metals with metal oxides. Analyzing the $\mathrm{T}_{50}$ temperature for the fresh monolithic catalyst, the Pd based catalyst was the most active $\left(\mathrm{T}_{50}=200{ }^{\circ} \mathrm{C}\right)$, whilst the metal oxides alone, $\mathrm{MnO}_{\mathrm{x}}$ and ${ }_{30} \mathrm{CuO}_{\mathrm{x}}$, were able to reach a conversion of $50 \%$ at 365 and $420{ }^{\circ} \mathrm{C}$, respectively. It was pointed out that impregnation of the metal oxides with Pd or Pt significantly decreased $\mathrm{T}_{50}$ for the resulting catalysts, however, they are still higher than those obtained in this study at least by $50{ }^{\circ} \mathrm{C}$. To conclude, all our monolithic catalysts 35 show $\mathrm{T}_{50}$ values around $150-160{ }^{\circ} \mathrm{C}$ after 3 cycles, indicating the benefits of the use of PVP polymer protected nanoparticles with more controllable properties. Furthermore, in our studies total oxidation of naphthalene is reached at lower temperatures.

Concerning the zeolite coated monoliths, a synergistic effect 40 between using zeolites (BETA or ZSM-5) prepared by an in situ methodology with PVP-Pd nanoparticles, has been found. The catalytic monoliths are able to produce total oxidation of naphthalene at low temperatures, together with naphthalene removal from the gas stream by adsorption at lower temperature

45 where the nanoparticles are not yet active. Secondly, the time-online experiments have identified that the catalysts are stable and thus should be regarded as suitable for the total oxidation of naphthalene in a practical application.

\section{Conclusions}

50 Zeolites BETA and ZSM-5, and silicoaluminophosphate molecular sieve, SAPO-5 have been coated on cordierite honeycomb monoliths (400 cpsi) by an in situ synthesis method. Two synthesis steps were required in order to coat the cordierite monoliths. The amount of zeolite introduced during the first 55 single-step synthesis cannot completely cover the monolith surface, but it is used to seed the second synthesis step, which produces homogeneous and continuous thin zeolite films.
Furthermore, the BETA coating layer is formed by a compact three-dimensional zeolite network conferring strong zeolite 60 anchoring to the cordierite structure. In general, the preparation reproducibility of the coated materials was very high.

All the coated monoliths were impregnated with Pd-PVPprotected nanoparticles and were used as catalysts for the total oxidation of naphthalene. All the catalysts showed high activity 65 for naphthalene conversion to $\mathrm{CO}_{2}$, with total conversion taking place at $165{ }^{\circ} \mathrm{C}$ after three cycles for Pd/MSAPO-5 and Pd/M $\gamma$ $\mathrm{Al}_{2} \mathrm{O}_{3}$ and $180{ }^{\circ} \mathrm{C}$ for Pd/MBETA and MSAPO-5 monoliths. All the catalysts possess high stability to temperature cycling, because their catalytic activity remained unchanged after testing 70 for several oxidation cycles. Time-on-line experiments have been used to test the stability of the catalysts. The Pd/MBETA and Pd/MZSM-5 samples were stable after accelerated ageing timeon-line for $48 \mathrm{~h}$ at $250^{\circ} \mathrm{C}$. However, despite being the most active after three cycles, $\mathrm{Pd} / \mathrm{SAPO}-5$ and $\mathrm{Pd} / \mathrm{M} \gamma-\mathrm{Al}_{2} \mathrm{O}_{3}$, exhibited 75 decreased catalytic activity for the conversion of naphthalene to $95 \%$, due to possible deactivation after ageing. It has been established that the use of Pd-based nanoparticles supported on BETA and ZSM-5 zeolites supported on honeycomb monoliths are very interesting options for the abatement of PAHs by 80 catalytic oxidation.

\section{Acknowledgements}

The authors would like to thank the Spanish Ministerio de Ciencia e Innovación, Generalitat Valenciana, and FEDER (Projects CTQ2012-31762 and PROMETEO/2009/047) for 85 financial support. F.J. Varela-Gandía thanks the University of Alicante for a PhD studentship. Á. Berenguer-Murcia thanks the Spanish Ministry for Economy and Competitiveness for a Ramón y Cajal fellowship (RyC 2009-03913).

\section{Notes and references}

$90{ }^{a}$ Departamento de Química Inorgánica e Instituto Universitario de Materiales, Universidad de Alicante, Ap. 99, E-03080, Alicante, Spain. Fax: +34 96590 3454; Tel: +34 96590 3946; E-mail: cazorla@ua.es ${ }^{b}$ Cardiff Catalysis Institute, School of Chemistry, Cardiff University, Main Building, Park Place, Cardiff CF10 3AT, UK. Fax: +44 29208 95 74030; Tel: +44 29208 74062; E-mail: TaylorSH@Cardiff.ac.uk

1 T. Garcia, B. Solsona, D. Cazorla-Amorós, Á. Linares-Solano, and S. H. Taylor, Appl. Catal. B, 2006, 62, 66

2 B. Puertolas, B. Solsona, S. Agouram, R. Murillo, A.M. Mastral, A. 100 Aranda, S.H. Taylor, and T. Garcia, Appl. Catal. B, 2010, 93, 395

3 A.M. Mastral, and M.S. Callen, Environ. Sci. Technol., 2000, 34, 3051

4 A. Aranda, S. Agouram, J.M. López, A.M. Mastral, D.R. Sellick, B. Solsona, S.H. Taylor, T.García, Appl. Catal.B, 2012, 127, 77.

1055 S. Scirèa, L.F. Liotta, Appl. Catal.B, 2012, 125, 222.

6 Z. Rui, Y. Lu, H. Ji, RSC Advances, 2013, 3, 1103.

7 C.L. Lin, Y.H. Cheng, J.Y. Chen, Journal of Hazardous Materials, 2011, 197, 254

8 E. Ntainjua N., and S.H. Taylor, Top. Catal., 2009, 52, 528

1109 C.C. Chang, C.Y. Chiu, C.Y. Chang, C.F. Chang, Y.H. Chen, D.R. Ji, Y.H. Yu, and P.C. Chiang, J. Haz. Mater., 161 (2009) 287-293.

10 L. Yu, X. Li, X. Tu, Y. Wang, S. Lu, and J. Yan, J. Phys. Chem. A,2010, 114, 360.

11 M.H. Yuan, Y.Y. Lin, C.Y. Chang, C.C. Chang, J.L. Shie, and C.H. 115 Wu, IEEE Trans. Plasma Sci., 2011, 39, 1092. 
12 S.C. Marie-Rose, T. Belin, J. Mijoin, E. Fiani, M. Taralunga, F. Nicol, X. Chaucherie, and P. Magnoux, Appl. Catal. B, 2009, 90, 489.

13 A. Bampenrat, V. Meeyoo, B. Kitiyanan, P. Rangsunvigit, and T. Rirksomboon, Catal. Commun., 2008, 9, 2349

14 X. Zhang, S. Shen, L.E. Yu, S. Kawi, K. Hidajat, and K.Y. Simon Ng, Appl. Catal. A, 2003, 250, 341

15 T. Garcia, B. Solsona, and S.H. Taylor, Appl. Catal.B, 2006, 66, 92

16 A. Aranda, E. Aylón, B. Solsona, R. Murillo, A.M. Mastral, D.R.

10 Sellick, S. Agouram, T. García, S.H. Taylor, Chem. Commun., 2012, 48, 4704.

17 L. Torrente-Murciano, A. Gilbank, B. Puertolas, T. García, B. Solsona, D. Chadwick, Appl. Catal.B, 2013, 132-133, 116.

18 D.R. Sellick, A. Aranda, T. García, J.M. López, B. Solsona, A.M.

15 Mastral, D.J. Morgan, A.F. Carley, S.H. Taylor, Appl. Catal.B, 2013, 132-133, 98.

19 T. Garcia, D. Sellick, F. Varela, I. Vázquez, A. Dejoz, S. Agouram, S.H. Taylor, B. Solsona, Appl. Catal. A, 2013,450, 169

20 J.-I. Park, J.-K.Lee, J. Miyawaki, W.-W.Pang, S.-H. Yoon, and I. Mochida, Catal. Commun., 2010, 11, 1068

21 A. K. Neyestanaki, L.-E Lindfors, T. Ollonqvist, and J. Väyryen, Appl. Catal. A, 2000, 196, 233

22 F.J. Varela-Gandía, Á. Berenguer-Murcia, D. Lozano-Castelló, D. Cazorla-Amorós, David R. Sellick, and Stuart H. Taylor, App. Catal.

$25 \quad B, 2013,129,98$

23 O. Öhrman, J. Hedlund, and J. Sterte, Appl. Catal. A, 2004, 270, 193

24 J.M. Zamaro, M.A. Ulla, and E.E. Miró, Chem. Eng. J., 2005, 106, 25

25 M.A. Ulla, R. Mallada, J. Coronas, L. Gutierrez, E. Miró, and J.

30 Santamaría, Appl. Catal. A, 2003, 253, 257

26 Jimmie L. Williams, Catal. Today, 2011, 69, 3

27 A. Bueno-Lopez, D. Lozano-Castelló, I. Such-Basáñez, J.M. GarcíaCortés, M.J. Illán-Gómez, and C. Salinas-Marinez de Lecea, Appl. Catal. B, 2005, 58, 1

3528 T.A. Nijhuis, A.E.W. Beers, T. Vergunst, I.Hoek, F. Kapteijn, and J.A. Moulijn, Catal. Rev., 2001, 43, 345380

29 Á Berenguer-Murcia, J. García-Martínez, D Cazorla-Amorós, Á Linares-Solano, and A.B. Fuertes, Micropor. Mesopor. Mater., 2003, 59, 147

4030 H. Lechert, R. Kleinwort, and H. Robson (Eds.), Verified Synthesis of Zeolitic Materials, 2nd ed., Elsevier, Amsterdam, 2001, pp. 198-200.

31 J.M. Campelo, F. Lafont, and J.M. Marinas, J. Catal., 1995, 156, 11

32 L. Villegas, F. Masset, and N. Guilhaume, Appl. Catal. A, 2007, 320, 43

4533 I. Miguel-García, Á. Berenguer-Murcia, and D. Cazorla-Amorós, Appl. Catal. B, 2010, 98, 161

34 D. Lozano-Castelló, F. Suárez-García, D. Cazorla-Amorós, Á. Linares-Solano, in: F. Beguin, E. Frackowiak (Eds),Carbon Materials for Electrochemical Energy Storage Systems, CRC Press,

$50 \quad$ Boca Raton (US), 2010, pp. 115-162.

35 J.M. López, M.V. Navarro, T. García, R. Murillo, A.M. Mastral, F.J. Varela-Gandía, D. Lozano-Castelló, A. Bueno-López, and D. Cazorla-Amorós, Micropor. Mesopor. Mater., 2010, 130, 239

36 V. Valtchev, J. Hedlund, B.J. Schoeman, J. Sterte, and S. Mintova, Micropor. Mater., 1997, 8, 93

37 J.M. Campelo, F. Lafont, J.M. Marinas, and M. Ojeda, Appl. Catal. A, 2000, 192, 85

38 J.M. Campelo, F. Lafont, and J.M. Marinas, Zeolites, 1995, 15, 97

39 M. Ferrandon, J. Carnö, S. Järås, and E. Björnbom, Appl. Catal.A, 1999, 180, 153 